\title{
Computing continuous nonlinear Fourier spectrum of optical signal with artificial neural networks
}

Sedov, Egor, Prylepskiy, Yaroslav, Chekhovskoy, Igor, Turitsyn, Sergei

Egor Sedov, Yaroslav Prylepskiy, Igor Chekhovskoy, Sergei Turitsyn, "Computing continuous nonlinear Fourier spectrum of optical signal with artificial neural networks," Proc. SPIE 11843, Applications of Machine Learning 2021, 118430J (1 August 2021); doi: 10.1117/12.2594127

SPIE Event: SPIE Optical Engineering + Applications, 2021, San Diego, California, United States 


\title{
Computing continuous nonlinear Fourier spectrum of optical signal with artificial neural networks
}

\author{
Egor Sedov ${ }^{\mathrm{a}, \mathrm{b}}$, Jaroslaw Prilepsky ${ }^{\mathrm{a}}$, Igor Chekhovskoy $^{\mathrm{b}}$, and Sergei Turitsyn ${ }^{\mathrm{a}, \mathrm{b}}$ \\ ${ }^{a}$ Aston Institute of Photonic Technologies, Aston University, Birmingham, UK \\ ${ }^{b}$ Novosibirsk State University, Novosibirsk, Russia
}

\begin{abstract}
In this work, we demonstrate that the high-accuracy computation of the continuous nonlinear spectrum can be performed by using artificial neural networks. We propose the artificial neural network (NN) architecture that can efficiently perform the nonlinear Fourier (NF) optical signal processing. The NN consists of sequential convolution layers and fully connected output layers. This NN predicts only one component of the continuous NF spectrum, such that two identical NNs have to be used to predict the real and imaginary parts of the reflection coefficient. To train the NN, we precomputed 94035 optical signals. 9403 signals were used for validation and excluded from training. The final value of the relative error for the entire validation dataset was less than $0.3 \%$. Our findings highlight the fundamental possibility of using the NNs to analyze and process complex optical signals when the conventional algorithms can fail to deliver an acceptable result.
\end{abstract}

Keywords: Nonlinear Fourier transform, Neural network, Optical communication, Signal processing

\section{INTRODUCTION}

Within various factors limiting the performance of modern optical communication systems, the nonlinearity of the optical channel is widely accepted as being one of the most challenging factors degrading the performance of installed optical lines. ${ }^{1}$ In this regard, a variety of methods aimed to increase the throughput of communication lines are being actively studied. ${ }^{2,3}$ Among the multiple alternative techniques, the NFT-based signal processing and the optical transmission techniques operating with the so-called nonlinear Fourier (NF) modes modulated with our data, have recently attracted a great deal of attention. The benefits of using the NFT-based processing in optical communication arise due to the fact that the NFT renders the effective linearisation of the signal evolution within the idealised single-mode fibre model, where the propagation of light is well approximated by the nonlinear Schrödinger equation (NLSE). The challenges in the practical implementation of the NFT-based transmission methods are related to the deviations of the true optical channel from the NLSE model and the sensitivity of the system to such deviations, ${ }^{4,5}$ and by the presence of optical noise. ${ }^{6-9}$ We also note that the NFT can be used for the analysis of the optical signals content, ${ }^{10,11}$ and has recently been extended for the identification of solitonic components in other photonic applications, including lasers and microresonators. ${ }^{12-16}$

The exact form of the NFT operations (both direct and inverse) associated with the focusing (anomalous dispersion) NLSE was first given in the seminal work by Zakharov and Shabat. ${ }^{17}$ The direct NFT corresponds to matching an optical signal with its nonlinear spectrum consisting, where the latter in the general situation can consist of discrete and continuous parts, ${ }^{18}$ though each part can be absent for some specific situations. A set of discrete eigenvalues corresponds to the soliton (discrete) part of the signal's NFT spectrum, which typically exists in the case of sufficiently high signal powers. ${ }^{11}$ However, the continuous part of the NFT spectrum, corresponding to the dispersive components, has proven to be very efficient in optical transmission applications. ${ }^{4,9,18,19}$

At present, a large number of "conventional" numerical methods have been proposed for the computation of nonlinear spectrum, ${ }^{20-23}$ and significant progress has been achieved in reducing the complexity of the algorithms $\left(\right.$ fast NFT) ${ }^{24}$ and in improving their accuracy. ${ }^{25-27}$ However, when applied to complex signals with a lot of nonlinear components, some difficulties referring to the stability issue can often emerge. ${ }^{28}$ Also, the real-time

E.S.: E-mail: e.sedov@g.nsu.ru

Applications of Machine Learning 2021, edited by Michael E. Zelinski, Tarek M. Taha

Jonathan Howe, Proc. of SPIE Vol. 11843, 118430J · (c) 2021 SPIE

CCC code: $0277-786 \mathrm{X} / 21 / \$ 21 \cdot$ doi: $10.1117 / 12.2594127$

Proc. of SPIE Vol. 11843 118430J-1 
NFT-based processing of complex waveforms is a difficult task, which limits our ability to implement NFT efficiently at the hardware level.

A promising area, in this case, is the use of machine learning (ML) and, in particular, the neural networks $(\mathrm{NN})$ based implementation of NFT. In recent years, there has been a big leap in the development of ML methods for solving algorithmically complex problems, such as, for example, pattern recognition and classification. ${ }^{29,30}$ The main stages here are training the model based on a set of some given data and applying the model to obtain a prediction. The first stage may take longer, but the application of the trained model is usually much faster, which makes it possible to implement systems based on ML methods on various unproductive devices. We note that the ML-related methods have been successfully used in compensation of nonlinear fiber and component-induced effects. ${ }^{31-34}$

In recent years, the ML has also been proposed to be used in NFT-based data transmission systems, but mostly for computing the soliton (discrete NFT spectrum) components. ${ }^{35-37}$ For the systems based on the continuous NFT spectrum, the NNs were used at the post-processing equalization stage ${ }^{38,39}$ There has been just one very recent work where a simple $\mathrm{NN}$ was used for the processing of continuous nonlinear spectrum: ${ }^{40}$ actually, the Matlab routine for the recognition of the hand-written digit was adapted there for the classification of constellation points, which brings about essential limitations for such an approach usage. In our work, we propose to implement a more advanced regression approach: we compute the continuous NFT spectrum using a special NN, and also restore the initial optical field (i.e. perform the inverse NN operation) using a special NN architecture.

\section{NONLINEAR FOURIER TRANSFORM}

The propagation of single light polarization in an optical fiber under some simplifying assumptions is well approximated by the NLSE, which, in a dimensionless form, is written as:

$$
i \frac{\partial q}{\partial z}+\frac{1}{2} \frac{\partial^{2} q}{\partial t^{2}}+|q|^{2} q=0,
$$

where, for simplicity, we have dropped out the deviations from the idealised model. Here $q(z, t)$ is the optical field evolving down the fiber, $z$ is the distance along with the fiber, and $t$ is the time variable in the reference frame co-moving with the group velocity of the light envelope. We do not include the nonzero gain-loss profile and noise terms as these are not important for our further considerations, but we note that Eq. (1) can also be understood as a path-averaged model approximating the non-zero gain-loss budget case. ${ }^{4,41}$

The NLSE written as in Eq. (1) belongs to the class of the so-called integrable equations. For our signal processing task in hand, it means that we can decompose the pulse propagation within the model (1) using the NFT operation in the bases of special nonlinear modes. We address the direct transformation, which consists in solving the so-called Zakharov-Shabat spectral problem for the localized "potential" $q(z, t)$, i.e. for our signal. The generally complex nonlinear spectral parameter $\lambda=\xi+i \eta$, entering this problem, can be understood as the nonlinear analogue of frequency. We write down the direct NFT as

$$
\left\{\begin{aligned}
-\partial_{t} \psi_{1}+q(z, t) \psi_{2} & =i \lambda \psi_{1}, \\
\partial_{t} \psi_{2}+q^{*}(z, t) \psi_{1} & =i \lambda \psi_{2},
\end{aligned}\right.
$$

where $\psi_{i}$ are some auxiliary functions. To determine the nonlinear spectrum associated with our localizes signal $q(t)$, we pick the special solution $\Phi(t, \lambda)=\left[\phi_{1}, \phi_{2}\right]$ of Eq. (2), imposing the special asymptotic condition at the trailing end of the pulse: $\Phi \rightarrow\left[e^{-i \xi t}, 0\right]$ when $t \rightarrow-\infty$. The core part of NFT is the calculation of scattering coefficients $a(\xi)$ and $b(\xi)$, defined through $\Phi(t, \lambda)$ as follows:

$$
\begin{array}{r}
a(\xi)=\lim _{t \rightarrow+\infty} \phi_{1}(t, \xi) e^{i \xi t}, \\
b(\xi)=\lim _{t \rightarrow+\infty} \phi_{2}(t, \xi) e^{-i \xi t} .
\end{array}
$$

The continuous spectrum $r(\xi)=b(\xi) / a(\xi)$ fills the real axis of the $\xi$-plane and corresponds to the dispersive wave component, being the direct analogue to Fourier transform and converging into it at low signal powers. The 
discrete part of the nonlinear spectrum is not addressed in our work here, and we further deal with the continuous part of the spectrum only. The signals are preselected in such a way that their associated NFT spectrum does not contain discrete levels, i.e. solitons, but we made sure that the nonlinear effects are significant, i.e. the continuous spectrum is noticeably different from its linear Fourier counterpart.

\section{BENEFITS OF USING NEURAL NETWORKS IN NONLINEAR SIGNAL PROCESSING}

Because the fibre-optic transmission systems involve simultaneously linear (memory), nonlinear, and noisy effects, they are very appropriate objects to be treated with the use of the latest advances in ML techniques. By using the ML we can solve the multidimensional optimization problem (referring, e.g., to the transmission quality and throughput maximization) without resorting to manipulations with all possible parameter values. The particularly relevant task is the identification of some internal features and patterns in the data, where the NNs can be used to model the propagation effects when our signal passes through the noisy nonlinear fiber medium. In other words, by using a NN, we can model a nonlinear transformation without the need for a direct calculation of that transformation. The gain lies in the speed and generality of this transformation, as well as in the flexibility and adaptability of the NN-based operations: the NN does not know what kind of data it processes, it searches inside the data for the necessary features that affect the final result and, then, extracts them. This process is called the feature extraction. So if we want to calculate a certain value of a function, then instead of (possibly) complex calculations we can use a pre-trained NN.

Another advantage of using NN-based signal processing is that the NNs can denoise the data. In practice, we always have some additional noise, which may be critical for idealized methods. A NN can filter out the excess information within itself, leaving only the main features necessary for a specific task. The possible disadvantage of using NN is their ultimate accuracy. However, in practice, the accuracy of $\mathrm{NN}$ is sufficient for most tasks and even exceeds the accuracy of existing numerical methods with a sufficient set of training data. As was noted before, the NNs have already demonstrated their decent potential in optical signal processing and channel equalization tasks, ${ }^{42}$ and, in particular, in the NFT-based systems. ${ }^{35-39}$

\section{RESULTS}

In this work, we use the NN to predict the continuous NFT spectrum of complex optical signals. For our study here we picked the example signals in the form of widely-used wavelength division multiplexing (WDM) format. A single return-to-zero WDM signal is represented as a sum of independent optical carriers:

$$
s(t)=\frac{1}{Q} \sum_{k=1}^{M} C_{k} e^{i \omega_{k} t} f(t), 0 \leq t<T,
$$

where $Q$ is the normalisation factor that we use to control the strenght of nonlinear effects, $M$ is the number of WDM channels, $\omega_{k}$ is the carrier frequency of the $k$-th channel, $C_{k}$ corresponds to the digital data in $k$-th channel, i.e. it is picked from some particular constellation, and, eventually, $T$ is the symbol interval. $f(t)$ is the waveform of a return-to-zero carrier pulse, which, in this work, without loss of generality, is taken (in the normalized form) as: $f(t)=\left[1-\cos \left(\frac{4 \pi t}{T}\right)\right]$ for $0 \leq t \leq \frac{T}{4}$ or $\frac{3 T}{4} \leq t \leq T$ and $f(t)=1$ for $\frac{T}{4}<t<\frac{3 T}{4}$. To assess the quality of the NN prediction, we use the following formula defining the relative error for the continuous NFT spectrum:

$$
\eta_{r}(\xi)=\frac{\left|r_{\text {predicted }}(\xi)-r_{\text {actual }}(\xi)\right|}{\left\langle\left|r_{\text {actual }}(\xi)\right|\right\rangle_{\xi}},
$$

where $\langle\cdot\rangle_{\xi}$ denotes the mean over the spectral interval, the "predicted" and "actual" indices refer to the NNpredicted and precomputed values of the reflection coefficient $r(\xi)$, respectively. We use $\left\langle\eta_{r}(\xi)\right\rangle_{\xi}$ to estimate the overall mean of the error. We stress that the metric was chosen in such a way as to take into account even the regions where the value of the spectrum or signal is much less than one.

Fig. 1a shows the architecture of the NN that performs the NFT operations (direct and inverse transform), with the parameters given inside the figure. The NN consists of sequential convolution layers and fully connected 


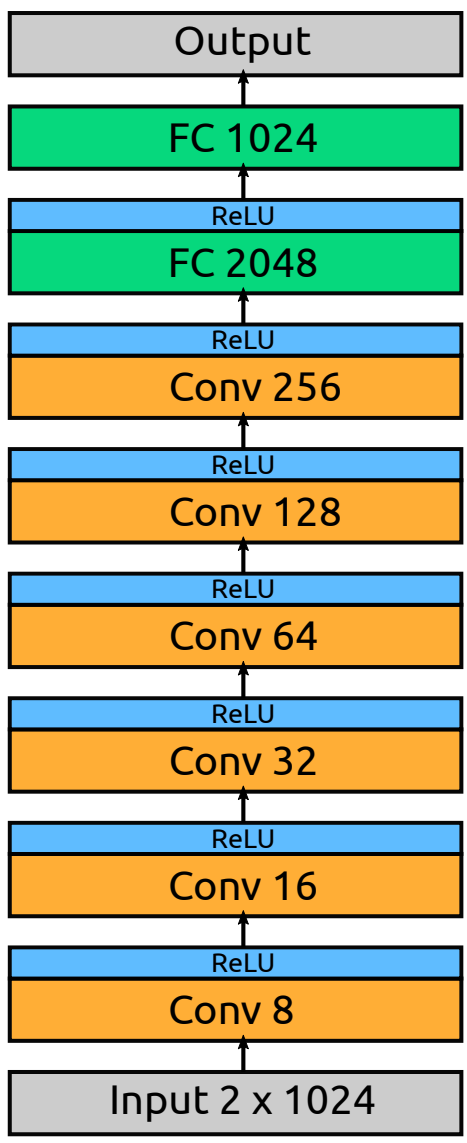

(a)

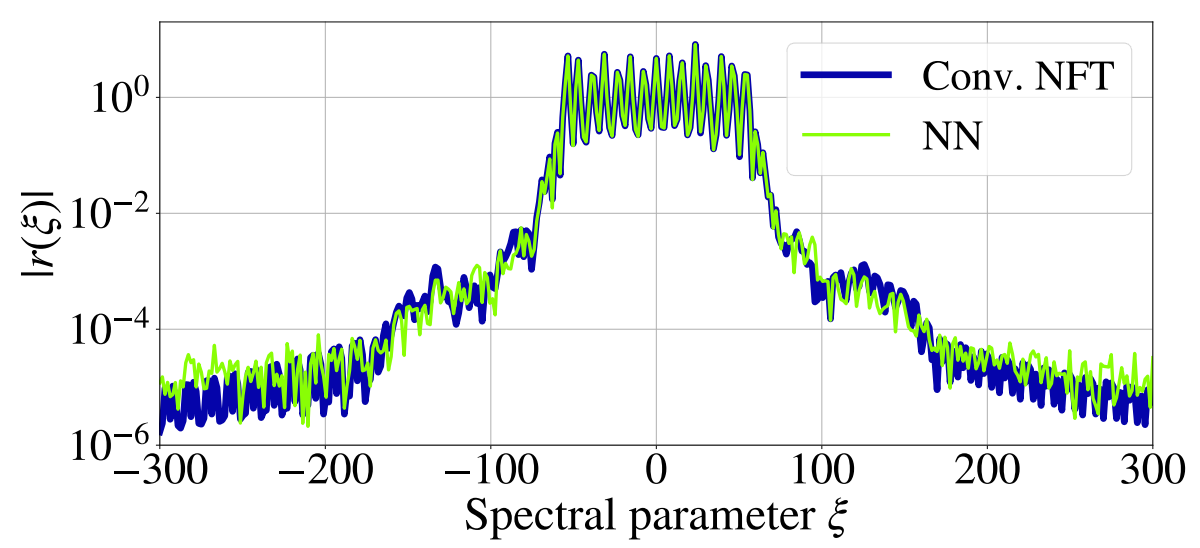

(b)

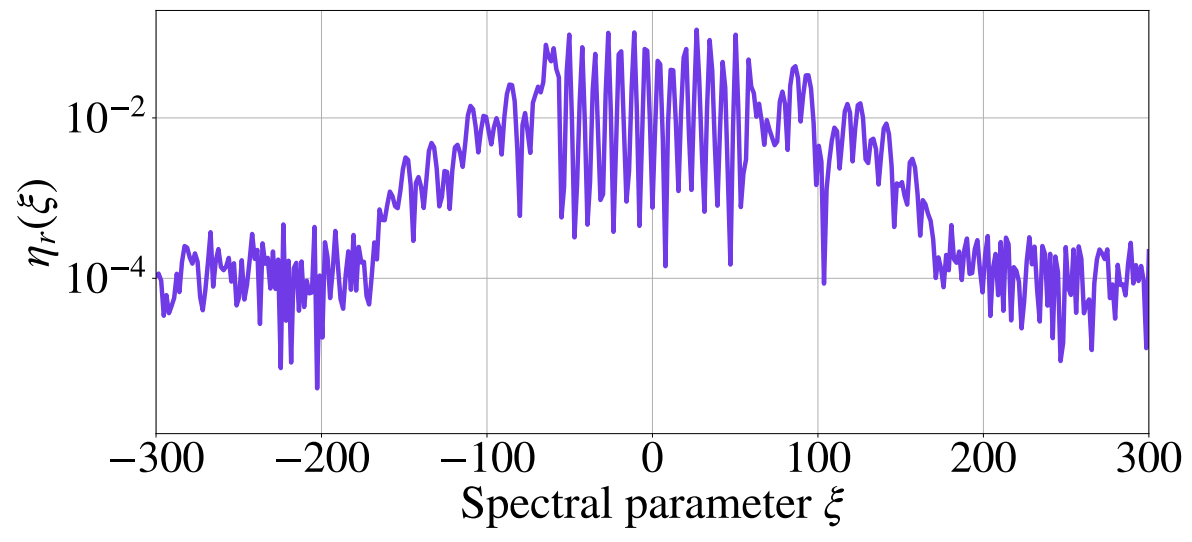

(c)

Figure 1: (a) Neural network architecture for predicting the continuous spectrum in a nonlinear Fourier spectrum for a complex signal. To predict the real and imaginary parts of the spectrum, we use two separate NNs with the same structures, but different weights. (b) Example of the amplitude of continuous nonlinear spectrum $r(\xi)$ calculated using the NN from (a) and with the conventional NFT method. The blue solid line shows the original spectrum. Green line show the NN predicted spectrum. (c) Example of metric (5) for continuous spectrum predicted by the NN from (a) trained on the WDM signals.

output layers. At the input, the network receives a complex signal consisting of 1024 points. This NN predicts only one component of the continuous NF spectrum, such that two identical NNs have to be used to predict the real and imaginary $r(\xi)$ parts. Each NN with the same architectures we trained independently.

There are 94035 signals in the dataset, 9403 are used for validation and are not involved in the training process. To generate the signals, we used random data sequences encoded in the Quadrature Phase Shift Keying (QPSK) format. The energy of all signals is the same and is chosen at such a level that nonlinear effects are strong. At the selected energy, some of the signals contained a discrete spectrum, but such signals did not get into the training dataset. The continuous spectrum for each signal had been precomputed using the conventional direct NFT methods. To train the NFT-Net, we used the mean squared error (MSE) as the loss function. In training the NFT-Net, we employed the Adam (Adaptive Moment Estimation) optimisation algorithm with a learning rate of $1 \mathrm{e}-4$. On average, with the amount of data used, our learning process took 50000 epochs.

Fig. 1c depicts $\eta_{r}(\xi)$ - the difference between the predicted and actual (precomputed using conventional NFT method) continuous nonlinear spectrum for the example signal. We see from the graph that NN performs forward transformation with high accuracy. Some increase in the error at the centre for a continuous spectrum is associated with its localization in the middle. While at the edges of the spectral interval, $r(\xi)$ values tend to 0 . The mean value over the entire validation set of the mean relative prediction error of the continuous spectrum 
$\left\langle\eta_{r}(\xi)\right\rangle_{\xi}$ for NN forward NFT is $2.68 \cdot 10^{-3}$. The results obtained show that the presented architecture can perform forward and backward NFT with high accuracy.

\section{CONCLUSION}

At the moment, ML and NN are state-of-the-art technologies that are actively researched in the fields of nonlinear signal processing and optical communications. The proposed NN architecture demonstrates the fundamental possibility of using the NNs to analyze and (de)modulate the complex optical signals used in communications. This opens up the prospects for improving existing systems without the need for a deep understanding of the internal nonlinear processes that affect the quality of signal transmission. We would like to stress that the method proposed in our current work is only the first step in the development of methods for machine processing of optical signals. It can be used to create smart receivers with digital back-propagation algorithms based on NFT and NN. Our findings demonstrate that the use of NN can allow studying not only the internal structure but also generating new signals using autoencoders. The fundamental possibility of using NN for NFT, in fact, can set up new areas for research related to the analysis of inherently nonlinear signal's structure and evolution characteristics. The next step in this direction can be the generalization of the results obtained in our work for the sake of our addressing the case of symbol sequences and simulating signal propagation in the NLSE-type channels.

\section{ACKNOWLEDGMENTS}

E.S. and I.C. acknowledges the support from Russian Science Foundation under Grant 17-72-30006 and the support by the grant of the President of the Russian Federation (MK-677.2020.9). E.S. and S.T. are supported by the EPSRC programme grant TRANSNET, EP/R035342/1. S.T. and J.P. acknowledge the support of Leverhulme Trust project RPG-2018-063.

\section{REFERENCES}

[1] Essiambre, R.-J., Foschini, G. J., Kramer, G., and Winzer, P. J., "Capacity limits of information transport in fiber-optic networks," Physical review letters 101(16), 163901 (2008).

[2] Winzer, P. J., Neilson, D. T., and Chraplyvy, A. R., "Fiber-optic transmission and networking: the previous 20 and the next 20 years," Opt. Express 26, 24190-24239 (9 2018).

[3] Cartledge, J. C., Guiomar, F. P., Kschischang, F. R., Liga, G., and Yankov, M. P., "Digital signal processing for fiber nonlinearities," Optics express 25(3), 1916-1936 (2017).

[4] Le, S. T., Prilepsky, J. E., and Turitsyn, S. K., "Nonlinear inverse synthesis technique for optical links with lumped amplification," Optics Express 23, 8317-8328 (2015).

[5] Yangzhang, X., Lavery, D., Bayvel, P., and Yousefi, M. I., "Impact of perturbations on nonlinear frequencydivision multiplexing," Journal of Lightwave Technology 36(2), 485-494 (2018).

[6] Derevyanko, S. A., Prilepsky, J. E., and Turitsyn, S. K., "Capacity estimates for optical transmission based on the nonlinear Fourier transform," Nature Communication 7(12710), 307 (2016).

[7] Civelli, S., Forestieri, E., and Secondini, M., "Why noise and dispersion may seriously hamper nonlinear frequency-division multiplexing," IEEE Photonics Technology Letters 29(16), 1332-1335 (2017).

[8] Civelli, S., Forestieri, E., and Secondin, M., "Mitigating the impact of noise on nonlinear frequency division multiplexing," Applied Sciences 10(24), 9099 (2020).

[9] Derevyanko, S., Balogun, M., Aluf, O., Shepelsky, D., and Prilepsky, J. E., "Channel model and the achievable information rates of the optical nonlinear frequency division-multiplexed systems employing continuous b-modulation," Optics Express 29(5), 6384-6406 (2021).

[10] Sedov, E. V., Redyuk, A. A., Fedoruk, M. P., Gelash, A. A., Frumin, L. L., and Turitsyn, S. K., "Soliton content in the standard optical ofdm signal," Opt. Lett. 43, 5985-5988 (12 2018).

[11] Turitsyn, S., Sedov, E., Redyuk, A., and Fedoruk, M., "Nonlinear spectrum of conventional ofdm and wdm return-to-zero signals in nonlinear channel," Journal of Lightwave Technology 38(2), 352-358 (2019).

[12] Sugavanam, S., Kopae, M. K., Peng, J., Prilepsky, J. E., and Turitsyn, S. K., "Analysis of laser radiation using the nonlinear fourier transform," Nature Communications 10(1), 5663 (2019). 
[13] Ryczkowski, P., Närhi, M., Billet, C., Merolla, J.-M., Genty, G., and Dudley, J. M., "Real-time full-field characterization of transient dissipative soliton dynamics in a mode-locked laser," Nature Photonics 12(4), 221 (2018).

[14] Turitsyn, S. K., Chekhovskoy, I. S., and Fedoruk, M. P., "Nonlinear fourier transform for characterization of the coherent structures in optical microresonators," Optics Letters 45(11), 3059-3062 (2020).

[15] Wang, J., Sheng, A.-G., Huang, X., Li, R.-Y., and He, G.-Q., "Eigenvalue spectrum analysis for temporal signals of kerr optical frequency combs based on nonlinear fourier transform," Chinese Physics B 29(3), 034207 (2020).

[16] Chekhovskoy, I., Shtyrina, O., Fedoruk, M., Medvedev, S., and Turitsyn, S., "Nonlinear fourier transform for analysis of coherent structures in dissipative systems," Physical Review Letters 122(15), 153901 (2019).

[17] Zakharov, V. E. and Shabat, A. B., "Exact theory of two-dimensional self-focusing and one-dimensional self-modulation of waves in nonlinear media," Soviet Physics JETP 34(1), 62 (1972).

[18] Turitsyn, S. K., Prilepsky, J. E., Le, S. T., Wahls, S., Frumin, L. L., Kamalian, M., and Derevyanko, S. A., "Nonlinear fourier transform for optical data processing and transmission: advances and perspectives," Optica 4(3), 307-322 (2017).

[19] Yousefi, M. and Yangzhang, X., "Linear and nonlinear frequency-division multiplexing," IEEE Transactions on Information Theory 66(1), 478-495 (2019).

[20] Delves, L. M. and Lyness, J. N., "A numerical method for locating the zeros of an analytic function," Math. Comp. 21, 543-560 (1967).

[21] Boffetta, G. and Osborne, A. R., "Computation of the direct scattering transform for the nonlinear schroedinger equation," Journal of computational physics 102(2), 252-264 (1992).

[22] Burtsev, S., Camassa, R., and Timofeyev, I., "Numerical Algorithms for the Direct Spectral Transform with Applications to Nonlinear Schrödinger Type Systems," Journal of computational physics 147(1), 166-186 (1998).

[23] Vasylchenkova, A., Prilepsky, J. E., and Turitsyn, S. K., "Contour integrals for numerical computation of discrete eigenvalues in the Zakharov-Shabat problem," Optics Lett. 43(15), 3690-3693 (2018).

[24] Wahls, S. and Poor, H. V., "Introducing the fast nonlinear Fourier transform," in [International Conference on Acoustics, Speech and Signal Processing], 5780-5784, IEEE, Vancouver (2013).

[25] Mullyadzhanov, R. and Gelash, A., "Direct scattering transform of large wave packets," Optics Letters 44, 5298 (11 2019).

[26] Medvedev, S., Chekhovskoy, I., Vaseva, I., and Fedoruk, M., "Conservative multi-exponential scheme for solving the direct Zakharov-Shabat scattering problem," Optics Letters 45, 2082 (4 2020).

[27] Medvedev, S., Vaseva, I., Chekhovskoy, I., and Fedoruk, M., "Exponential fourth order schemes for direct Zakharov-Shabat problem," Optics Express 28, 20 (1 2020).

[28] Gelash, A. and Mullyadzhanov, R., "Anomalous errors of direct scattering transform," Physical Review E 101, 052206 (5 2020).

[29] Bishop, C. M., [Pattern recognition and machine learning], springer (2006).

[30] Duda, R. O., Hart, P. E., and Stork, D. G., [Pattern classification], John Wiley \& Sons (2012).

[31] Zibar, D., Winther, O., Franceschi, N., Borkowski, R., Caballero, A., Arlunno, V., Schmidt, M. N., Gonzales, N. G., Mao, B., Ye, Y., et al., "Nonlinear impairment compensation using expectation maximization for dispersion managed and unmanaged pdm 16-qam transmission," Optics express 20(26), B181-B196 (2012).

[32] Zibar, D., Piels, M., Jones, R., and Schäeffer, C. G., "Machine learning techniques in optical communication," Journal of Lightwave Technology 34(6), 1442-1452 (2015).

[33] Sidelnikov, O. S., Redyuk, A. A., Sygletos, S., and Fedoruk, M. P., "Methods for compensation of nonlinear effects in multichannel data transfer systems based on dynamic neural networks," Quantum Electronics 49, 1154-1157 (12 2019).

[34] Sidelnikov, O., Redyuk, A., and Sygletos, S., "Equalization performance and complexity analysis of dynamic deep neural networks in long haul transmission systems," Optics Express 26, 32765 (12 2018).

[35] Jones, R. T., Gaiarin, S., Yankov, M. P., and Zibar, D., "Noise robust receiver for eigenvalue communication systems," in [Optical Fiber Communication Conference], W2A-59, Optical Society of America (2018). 
[36] Jones, R. T., Gaiarin, S., Yankov, M., and Zibar, D., "Time-domain neural network receiver for nonlinear frequency division multiplexed systems," IEEE Photonics Technology Letters 30(12), 1079-1082 (2018).

[37] Yamamoto, S., Mishina, K., and Maruta, A., "Demodulation of optical eigenvalue modulated signal using neural network," IEICE Communications Express 8(12), 507-512 (2019).

[38] Kotlyar, O., Pankratova, M., Kamalian-Kopae, M., Vasylchenkova, A., Prilepsky, J. E., and Turitsyn, S. K., "Combining nonlinear Fourier transform and neural network-based processing in optical communications," Optics Letters 45, 3462 (7 2020).

[39] Kotlyar, O., Kamalian-Kopae, M., Pankratova, M., Vasylchenkova, A., Prilepsky, J. E., and Turitsyn, S. K., "Convolutional long short-term memory neural network equalizer for nonlinear fourier transformbased optical transmission systems," Optics Express 29(7), 11254-11267 (2021).

[40] Zhang, W. Q., Chan, T. H., and Afshar, S., "Direct decoding of nonlinear ofdm-qam signals using convolutional neural network," Optics Express 29(8), 11591-11604 (2021).

[41] Kamalian, M., Prilepsky, J. E., Le, S. T., and Turitsyn, S. K., "On the design of nft-based communication systems with lumped amplification," Journal of Lightwave Technology 35(24), 5464-5472 (2017).

[42] Freire, P. J., Osadchuk, Y., Spinnler, B., Napoli, A., Schairer, W., Costa, N., Prilepsky, J. E., and Turitsyn, S. K., "Performance versus complexity study of neural network equalizers in coherent optical systems," arXiv preprint arXiv:2103.08212 (2021). 